\title{
EFFICIENT DATA GATHERING IN SUPPORT OF DESIGN ISSUE RESOLUTION IN AN AUTOMOTIVE COMPANY
}

\author{
T. M. Sissoko ${ }^{1,3, \bowtie}$, M. Jankovic ${ }^{1}$, C. J. J. Paredis ${ }^{2}$ and E. Landel ${ }^{3}$ \\ ${ }^{1}$ CentraleSupélec, France, ${ }^{2}$ Clemson University, United States of America, ${ }^{3}$ Renault, France \\ $\square$ timothe.sissoko@centralesupelec.fr
}

\begin{abstract}
When designing complex systems, multiple people contribute to the process of information collection in support of decision making. In this paper, we study information collection in the Issue Resolution Decision Support (IRDS) framework. We assess the difficulties associated with uncertainty in the often scarce data when implementing the framework in a company and map out how the data sources are scattered across the organization. We study the elicitation process and propose to leverage sensitivity analysis to better allocate data collection efforts.
\end{abstract}

Keywords: uncertainty, decision making, knowledge sharing, data collection, decision support

\section{Introduction}

When addressing design decisions for the development of complex systems, decision-makers often face situations characterized by uncertainty (Rocquigny et al., 2008). This uncertainty is both related to the design artifact and the design process that can be impacted by interrelated decisions. However, until recently, the vast majority of research in decision-based design (DBD) (Chen et al., 2013) focused decision problems formulation on the design artifact and overlooked the tradeoffs involved when gaining additional information. To expand this scope, Thompson and Paredis (2010) established a new DBD perspective that represents the tradeoffs under consideration when analyses can be performed to incorporate additional information in the design phase. They show that the decision analysis of design process decisions provides a more comprehensive model of the problem when multiple information sources can sequentially be used.

In the context of decision-making addressing design issues, Thompson and Paredis' process-focused approach has been extended in a model that integrates concurrent analyses, impacts of potential changes related to the product definition evolution and project time constraints. Further details of the model are provided in the reference (Sissoko et al., 2019). IRDS (Issue Resolution Decision Support) framework has been proposed to operate this model in an enterprise context. The authors highlighted that data gathering can be challenging, costly, and that biases can be introduced by the elicitation and modelling of experts' beliefs. The implementation of a decision support framework in a company implies to tackle difficulties and uncertainties related to the prioritization of data providers, the elicitation and modeling of their beliefs, and the representation and the interpretation of the results provided by the decision analysis. Managing the uncertainty when implementing IRDS in a company setting is necessary to perform valuable decision analysis and help the project team to gain insight and take actions. Sensitivity analysis has been used along with decision analysis in the literature (Howard 
and Abbas, 2015) to help to focus data-gathering effort (Laskey, 1995; Leurent et al., 2018). In the context of IRDS, sensitivity analysis is worth studying from a cost-efficient data collection perspective. In this respect, the paper aims to address the following question:

What are the difficulties related to the uncertainty encountered when implementing IRDS and how to manage them?

As we conducted our research, this general question leads to the following specific questions:

- How to incorporate various experts' beliefs that are sometimes contradicting each other and expressed in different forms in a numerical model?

- How can sensitivity analysis influence data collection strategy?

The study in this paper has been conducted in a multinational automotive company. It is part of a research project that aims to support decision making for resolving design issues. This project includes an empirical study (Sissoko et al., 2018) and the creation and test of the decision support framework IRDS (Sissoko et al., 2019).

In Section 2, we present the methodological framework of the research project and provide further details on the methodology used in the current study. In Section 3, we discuss the uncertainty and the related difficulties encountered when implementing IRDS framework. In Section 4, we introduce a sensitivity analysis-based method which aim to address these issues. We end with conclusions and future work in Section 5.

\section{Methodology}

The research presented in this paper corresponds to the last part of a research project in which the methodological framework has been strongly inspired by the Design Research Methodology (Blessing and Chakrabarti, 2009), as illustrated in Figure 1.

$\begin{gathered}\text { Macro description } \\ \text { of the industrial } \\ \text { problem }\end{gathered}$
$\begin{gathered}\text { Data collection and } \\ \text { analysis }\end{gathered}$
Descriptive study
Sissoko et al., 2018

Figure 1. Methodological framework of the research project

The paper focuses on the identification of the implementation challenges and propositions of improvements. Hence, this study is both descriptive and prescriptive. We used the research material produced in a previous descriptive study (Sissoko et al., 2018), which was based on the collection and analysis of data from three sources: company documents, interviews of 11 stakeholders, and 40 decision problems observed in decision meetings. These allowed us to understand the as-is decisionmaking and simulation processes and to identify the challenges encountered by the industry professionals. The empirical study enabled us to define specifications for a decision support framework that we created and tested together with experts in the company through a prescriptive study. The decision support framework, based on normative decision theory, is introduced and detailed in (Sissoko et al., 2019). For To better identify the challenges of decision support framework implementation, the present study first compares the information already available in as-is decision dossiers and the information that is required to use IRDS. To do so, we extend the observation to 45 decision dossiers brought to project manager level decision meetings and interviewed 15 more industry professionals. The five additional decision dossiers have been used as case studies for the implementation of IRDS.

Semi-structured interviews conducted by one researcher were analysed and cross-checked with two other members of the research team. Questions were designed to understand the as-is decisionmaking process and data gathering. For the present research, we included new interviewees and focused questions on data-gathering. For example: "What data are communicated in decision dossiers?", "Where do these data come from?", "How long does it take and how much does it cost to produce such data?". 
Furthermore, we tested the decision support framework on five case studies which correspond to real industrial problems. Through iterative meetings with individual from various positions (simulation analysts, CAE synthesis engineers, architects, etc.), we estimated the difficulty to obtain input data and devised the best ways to obtain these data with valuable accuracy and at reasonable cost. More details about the case studies are provided in (Sissoko, 2019).

\section{The uncertainty associated with gathering data}

Implementing a decision support framework in a company implies dealing with the actual company processes, practices, and to a bigger extent, its culture. The introduction of new methods and tools impact the current practice and organization and vice versa (Aladwani, 2001; Jones et al., 2006; Matt et al., 2015). The objective was to identify the challenges of implementation from an operational perspective, so that the framework can be best integrated in the company without necessary transforming abruptly the organization and practices. We focused the research on the information collection for supplying IRDS' model inputs.

\subsection{Available data and access to information in company settings}

We identified what type of information and through which means it is conveyed in decision meetings. There are three communication means:

Decision dossier presentation documents: These are a PowerPoint documents composed of a dozen slides either presented by the Synthesis Architect, the Customer Performance Leader, the CAE Synthesis Engineer, or any knowledgeable person on the subject. It presents the issues and the artifact alternatives - also called hypotheses at the OEM - to solve it. It synthetizes the stakes, performance, cost and time data related to the design issue framed as a decision problem.

Quotes from decision meeting participants: These exchanges aim to enrich the knowledge of the participants and, ultimately, influence the decision maker. Such quotes can relate to the simulation results or cost estimates presented, including the expression of uncertainty.

Water-cooler talk: These are discussions held aside from decision meetings where implicit knowledge and data that are not included in the presentation can be shared.

Figure 2 presents the roles that supply the information that is available in current decision dossiers. The fact that data included in decision dossiers may be supplied by various roles will be relevant when we will establish a parallel with the data gathering of IRDS inputs. For instance, the costs can be estimated by both designers (through heuristics), cost analysts (through cost simulation and further analyses), Engineering Leaders (who works closely together with designers, supplier, and manufacturer) and synthesis architects (through heuristics or gathering and interpreting other roles' estimates). The same applies to performance estimates with the corresponding roles. The timing data are seldom provided explicitly with regard to design process alternatives (analyzing further a solution, time between one decision meeting and another, etc.). However, the project team can agree on an estimate of the delay in the manufacturing of prototypes caused by the choice of a technical solution.

We observed that $5 \%$ to $20 \%$ of IRDS inputs are already available in the current decision dossiers (vendor tooling cost, per-unit manufacturing cost, weight variation, $P(O K \mid \overline{I T D C})$, see section 3.2.1) this proportion varies because some decision dossiers do not contain enough information to compare technical solutions with each other. The Probability of Success is seldom communicated directly as a numerical probability. It can rather be inferred from a Boolean representation (OK/NOK) or a smiley rating scale (sad-red / sad-orange / happy-orange / happy-green). Due to subjective and therefore variable definitions and mental models, the inference and translation of performance data, qualitative ratings, and personal beliefs into numerical probabilities can be ambiguous. Other information that is lacking in current decision dossiers needs also to be elicited and translated into IRDS input data.

It is important to note that to date, the information contained in decision dossiers, and ultimately presented in decision meetings, only consider the scenario of success. It means that the costs considered only reflect the costs that will be committed if no rework is needed. The costs involved in case of failure are not explicated (e.g. corrective design, changing tooling, etc.). A corollary of this framing choice is that risk is not represented. Indeed, the probability of success - and its opposite - is 
not explicitly considered. The information also does not contain the evolution of the product and unknown changes considerations. It means that the way the decision problem is framed does not include the likelihood that changes may occur and affect the decision outcomes.

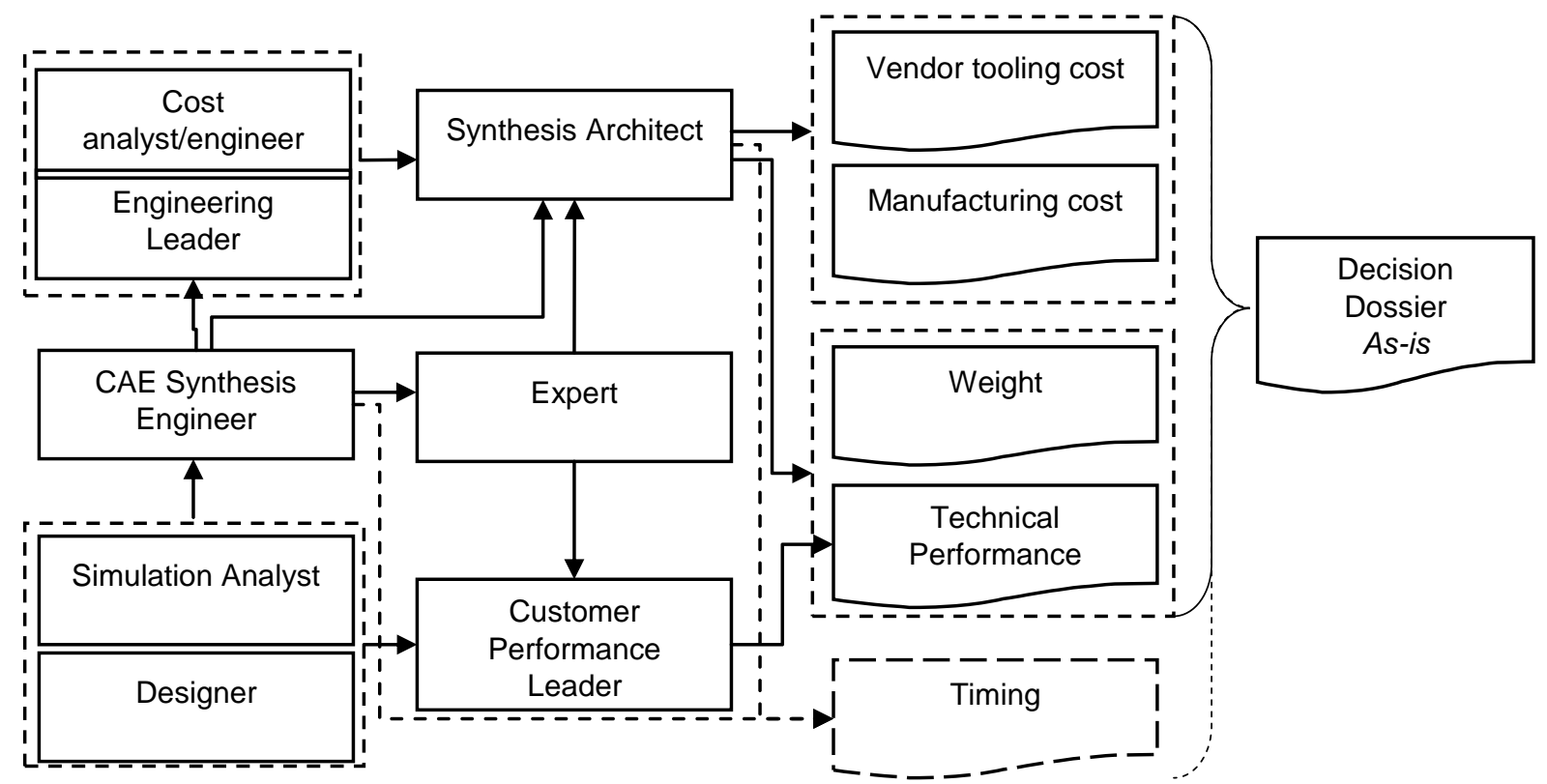

Figure 2. Information sources in as-is decision dossiers

IRDS aims to provide a more comprehensive framing of decision problems. It computes the expected utility by incorporating costs involved in case of failure, probability of success/failure, and changes in the decision situation. Computing requires input data, and this is where it can become a challenge in an enterprise context. This leads us to consider the notion of belief modeling.

\subsection{Modeling data providers' beliefs}

Data providers traditionally communicate their beliefs in decision dossiers in the form of written sentences associated with plots and 3D simulation screenshots, numerical values and smiley faces. These representations usually serve as basis for discussion between participants of the decision meetings.

When considering enhancing the information in a decision analysis perspective, these qualitative and quantitative representations of data providers' beliefs can be leveraged to infer quantities such as the probabilities of success and the costs (e.g. from "this solution is more expensive" to "likely $2 €$ more expensive"). In the current practice, statistical data are seldom provided for technical performance since it involves performing important numbers of simulations, which is time consuming and costly (durability performance and advanced driver-assistance systems are some exceptions). Simulations are usually done in relatively small numbers and integrate nominal parameters - i.e. seldom take into account variability due to changing design specifications and manufacturing process deviations.

\subsubsection{Probabilities of success}

Let us examine the input $\boldsymbol{P}(\boldsymbol{O K} \mid \overline{\boldsymbol{I T D C}})$. This input is a probability that reflects the chance that once the technical solution is incorporated in the vehicle, assuming that no changes will impact the decision, it will lead to a success when the prototype of the vehicle is tested in the manufacturing phase. One considers the state "success" if the performance target is met. In the current practice, information of various nature concerning the performance of a technical solution is provided to the decision-maker. In our empirical study, decision-makers indicated that they formulate their own beliefs about the chances of success of a technical solution based on the numbers, verbatims of participants, etc. and by confronting them with their own experience and "intuition". Experts proceed the same way when they report their beliefs in the form of verbatims. $P(O K \mid \overline{I T D C})$ takes a single value to capture the prediction about a binary event. For such a belief representation, a simple and 
time-efficient rule has been used in the literature of belief elicitation: the free rule (Hollard et al., 2010). With this rule the decision analyst simply ask the experts to report their beliefs - without confronting them with an incentive mechanism. This elicitation can include the translation or interpretation of the beliefs representations already provided (such as smiley faces) by other data providers. This translation is made together with the decision analyst. It is useful from a knowledge capitalization perspective (capturing information from historical decision problems and performing analyses with ex-post information).

Experts translate the smiley faces already existing in decision dossiers into $P(O K \mid \overline{I T D C})$ as follow: between $0 \%$ and $25 \%$ for sad-red, between $25 \%$ and $50 \%$ for sad-orange, between $50 \%$ and $75 \%$ for happy-orange, between $75 \%$ and $100 \%$ for happy-green. These assumptions are made in accordance with the data providers interviewed; they assumed a variation on the responses depending on the problem considered, but also depending on the experts' subjectivities. For sad-red and happy-green, we observed in the case studies that experts tend to assess $P(O K \mid \overline{I T D C})$ that are closer to the extrema ( $0 \%$ or $100 \%$ chances of success). This can be explained by the fact that they are more comfortable with translating what they consider as strong statements, such as "sad-red means that there is very few chances to pass, therefore I would give a little 5\%, even less" or "happy-green means that it is ok, I would give 95\%, even more". The drawback of this approach is that the elicitation can be biased by the fact that experts assess a probability through a model that already is already an interpretation. Moreover, more effort is needed to combine the smiley faces across several disciplines to come to an overall estimate. A direct approach, consisting in eliciting the belief without the support of smiley faces could avoid this bias - we did elicit without smiley faces, but the comparison of the two approaches for the same case studies is not included in the ongoing research.

Let us now consider $\boldsymbol{P}(\boldsymbol{O K} \mid \boldsymbol{I T} \boldsymbol{D C})$. This probability is similar to the latter, with the difference that impacting changes are assumed to occur. This input variable is more challenging to elicit since it involves additional assumptions strongly related to the experience of the experts. For instance, some changes are known to be likely occur at specific milestones. In accordance with experts, we assumed that a default value of $P(O K \mid I T D C)$ is $50 \%$ (reflecting ignorance) for technical solutions that have $P(O K \mid \overline{I T D C}) \geq 50 \%$ (i.e. the vast majority of the technical solutions that are considered to solve a design issue).

IRDS proposes a simple model for $P(O K \mid I T D C)$ and supposes that changes have negative impacts in accordance with experts interviewed - even if it is not always true. Predicting whether the changes will impact positively or negatively the performance of the technical solution considered is more challenging. The impacts of changes can also change overtime. For instance, the changes that occur before a certain moment can have positive impact, and the changes occurring after can have negative impact. In that respect, $P(O K \mid I T D C)$ can be time dependent. However, it is even more difficult to elicit beliefs for such a model since it requires additional assumptions not necessarily supported by empirical evidences. IRDS still enables the user to compute the problem with time-dependent $P(O K \mid I T D C)$ if enough information is available.

Both $P(O K \mid \overline{I T D C})$ and $P(O K \mid I T D C)$ are conditional to $\boldsymbol{p I T D C}(\boldsymbol{t})$. This function represents the probability that later or current but not represented changes in the technical definition of the vehicle will impact the outcomes of the current decision. Its values depend on the design issue itself: whether the system under consideration is isolated or interacting with others, and whether the interactions affect the performances under consideration. Defining $\operatorname{PITDC}(t)$ from empirical evidence is so far very difficult. It would imply to, at least, gather data about design modifications on a significant number of similar projects over time, and relate these design modifications to each other with regards to the technical performance under consideration to formulate assumptions based upon objective observations. Ideally, a synthesis model or influence diagram associated with historical data would help one to define $\operatorname{pITDC}(t)$. These supports would involve investing in knowledge management systems development, which is the tendency in a digital transformation perspective, and the work towards this direction was not mature when we performed this research. However, experienced simulation practitioners who are among the first persons informed of design changes can formulate assumptions and provide $p I T D C(t)$ profiles. These educated guesses are easy to elicit with a free rule. Simply asking them "at this time, what are the chances that changes that impact the performance 
measured occur?" allows the decision analyst to collect values - cartesian coordinates used to build the function. A short iterative elicitation process where the data provider can readjust the profile of the function is sufficient to obtain an estimate of $\operatorname{PITDC}(t)$ in less than $10 \mathrm{~min}$. In the example shown in Figure 3, the data provider (a Simulation Analyst) reported his beliefs about $p I T D C$, for a specific part, in function of the development process milestones - and the design specifications that are generally set at these moments.

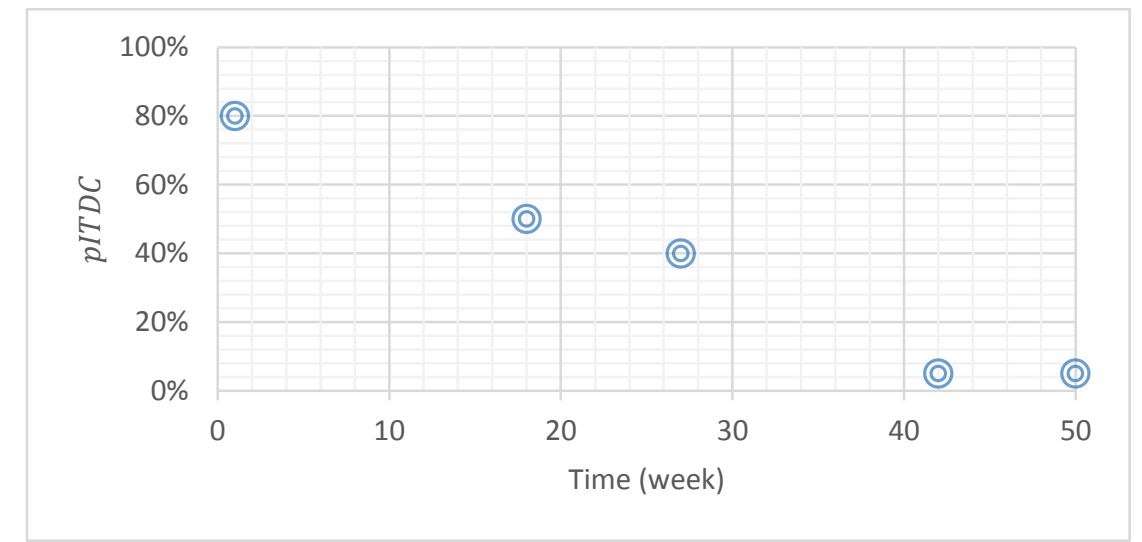

Figure 3. Example of elicited pITDC coordinates in accordance with development process milestones

\subsubsection{Costs}

IRDS' cost structure include costs committed during and related to the development phase, costs committed during the development phase and related to the industrialization phase, costs committed during and related to the industrialization phase, and a cost of delay function.

The costs estimates can be supplied by different actors, as shown in Figure 2, with different precision and level of confidence. For instance, for industrialization costs, a synthesis architect can provide costs estimates based on heuristics and rules that do not really reflect the actual suppliers negotiated prices or specific manufacturing processes. Cost analysts perform simulations with a large number of inputs of various nature to support purchasers who negotiate with suppliers. Both the simulations outputs and negotiated prices can be used as estimates in as-is decision dossiers. In the fact, some interviewee reported that the persons who enter the costs estimates can deliberately choose to reveal one value over another to influence the decision-maker for hidden interests.

Of course, it is difficult to provide costs estimates based on negotiated prices for newly designed technical solutions since the issue resolution process has to lead to the selection of a technical solution that will be used as an input for negotiations with suppliers. However, historical data of negotiated prices for similar technical solutions in similar project can help to infer costs estimates. For costs related to vendor tooling or supplier engineering studies, some estimates based historical data are used as references. Considering this setting, IRDS proposes to integrate probability distributions to represent the uncertainty of data providers regarding costs. We observed that, when interviewed, data providers spontaneously provide a variation range associated with a value that they consider as the most probable rather than providing a single value - like they do in the decision dossiers. This threepoint estimation represents the less expensive, most likely, and most expensive estimates, and is convenient to build a triangular distribution.

As mentioned above in Section 3.1, only the costs engaged in case of success are provided in the current practice. Although the data providers traditionally do not explicit their beliefs about the costs incurred in case of failure, they do not struggle make assumptions about these costs when they have a backup plan in mind. For some design issues causing appearing in the manufacturing phase, Simulation Analysts and designers know what type of solution is more likely to be implemented, and what procedures it involves (e.g. designing a reinforcer, changing the tooling, manufacturing a new prototype, etc.). The costs associated with these interventions are estimated the same way as in the scenario of success, but involve to discuss further the assumptions. We observed that this effort help 
the data provider to anticipate the rework needed and related expenses, and consider risk more explicitly.

The penalty and added values quantities can be related to the cost of additional weight (established by the management control and straightforward to provide), the savings due to reusing tooling, etc. and are supplied as the costs mentioned above. It can be challenging to quantify these inputs when it comes to taking into consideration other projects that may be impacted by the decisions made for the one under consideration.

The cost of delay is a function that represent the cost incurred by the delay in the project over time. Due to a lack of measurements and tracking of delay, this function is difficult to define precisely. However, experts assume it to have an exponential profile. They consider the penalty related to postponement of the production launch - the management control provides estimates about the daily loss in such situations - and the increase of full-time equivalent staff requested in order to meet the deadlines. The latter consideration depends on the nature and the quantity of activities that depend on the design issue to be solved. The decision analyst can build $\operatorname{Cost}_{\text {delay }}(t)$ by eliciting coordinates with a free rule in the same fashion as $p I T D C(t)$. As some problems are similar and are detected at similar moments in the development process, $\operatorname{Cost}_{\text {delay }}(t)$ reference profiles can be established over time and experience.

\subsubsection{Accuracies of the analyses for technical solutions}

The additional analyses can be either related to numerical simulations, early physical tests (when possible), studies conducted by suppliers, or experts judgments. The input variables that indicate the accuracy of the additional analyses are the sensitivity (true positive rate) $P(F a v \mid O K)$ and the specificity (true negative rate) $P(\overline{F a v} \mid \overline{O K})$. When considering the most common information source, i.e. numerical simulation, the simulation practitioners and methods specialists do not characterize the accuracy of numerical simulation analyses with true positive and true negative rates. Since only design specifications with favorable analyses results are normally brought to the manufacturing phase, correlation studies performed between numerical models and physical tests measurements and feedback from the manufacturing phase can provide insight to assess $P(F a v \mid \overline{O K})$. The specificity is more difficult to assess with this approach. However, data providers are asked to define these input variable with respect to the confidence they have into the processes that provide them additional information. Hence they provide estimates of these values through an free-rule elicitation.

\subsubsection{Analysis times}

Whether considering numerical simulation or other information source, in the current practice, simulation practitioners or other expert provide the time that is necessary to obtain informative results, and the project manager defines, on the basis of this estimate, at which meeting date the issue will be re-examine with new information. This difference between two decision meetings corresponds to At. Hence, in some situations, the project manager - who takes on the role of decision-maker - can also be a data provider. Knowing the decision meetings are held weekly, the decision analyst can easily define with data providers the value of $A t$.

In conclusion, the decision analyst has to model data providers' beliefs when gathering data to use IRDS. He or she has to identify the data provider that is the most likely to supply accurate data, make a tradeoff between the time necessary to complete the elicitation process and the accuracy sought, and foster the commitment of data providers to carry out additional work. This is particularly the case in the early phase of implementation - the phase we focused our research on - in a situation where the new practices are not yet established.

\section{Sensitivity analysis-based method}

When testing the framework on historical design issues, it was difficult to enhance the available information through an elicitation process in a timeframe consistent with our project. Indeed, some of the individuals who are the more knowledgeable about certain data were unavailable. That led us to assume estimates together with non or less-expert data providers for some input data. We performed 
sensitivity analyses to examine whether seeking for more accuracy was actually necessary. Our observations prompted us to reconsider the data gathering strategy, as discussed in the next sections.

\subsection{Model type: deterministic or probabilistic}

The decision problem model can be either considered as deterministic or probabilistic (Iooss and Lemaitre, 2015). We investigated two approaches to perform a sensitivity analysis on a decision problem with IRDS.

In the deterministic approach, input variables are nominal values and are varied one at a time. This approach is convenient when we gather data gather from existing information sources - i.e. as-is decision dossiers. This approach leverages sensitivity analysis to account for uncertainty. Sensitivity analysis yet requires determining variation ranges that should be consistent with data provider's uncertainty. Hence, an initial elicitation effort is necessary for each type of input, at least to formulate assumptions for common variation ranges. In the theoretical case where it would be impossible to elicit variation ranges, or the decision analyst wants to explore a problem briefly, we found reasonable to use $20 \%$ as a common variation ranges for costs, and a standard logistic function for the bounds of the probabilities of success ranges. As discussed in Section 3.2, tackling decision problems with this approach upfront can help to determine how a complementary probabilistic approach should be handled.

In the probabilistic approach, inputs are provided in the form of probability distributions, after an elicitation process, as discussed in Section 3.2.1. In this case, the distribution parameters are varied, and not only the nominal value of the input. This allows the analyst to identify which input variation is likely to change the decision, and to determine whether further analyses should be performed on the beliefs modeled. Performing a sensitivity analysis with probabilistic distributions requires numerical tools and methods (e.g. Monte Carlo simulations, etc.) and is more resources consuming than the deterministic approach.

In Section 3.2.2. we stated that the simplest distributions used as cost inputs are triangular distributions. These distributions account for the most probable value (the mode) and upper and lower bounds. In the particular case of symmetric triangular distributions, the mean actually corresponds to the mode. In this case, we think it is reasonable to use a variation range of $20 \%$ both for the variation of the mode and the variation of the lower and higher bounds. The same logistic function is used for the variation of the probabilities of success.

We observed that when considering a linear model - i.e. static in the case of IRDS, at a given $t$ - the EV results tend to be similar whether symmetric triangular distributions are provided with the deterministic approach or nominal values are provided with the deterministic approach. The underlying assumption is that the deterministic-nominal values entered correspond to the probabilisticmeans of triangular distributions. Hence, in this case, a sensitivity analysis based on a deterministic approach leads to valuable results at a reasonable cost.

\subsection{Impacts on the data gathering process}

Analyzing a decision problem with IRDS requires to gather information related to a set of alternatives and to perform a computation that issue an expected value for each alternative. The preferred alternative is thus identified and communicated to the decision-maker. We first considered rigorously collecting accurate input data, in order to accurately calculate the expected values and thus be able to correctly distinguish the outcomes of the alternatives while ranking them. We observed that an exhaustive and detailed information gathering process is both cognitively demanding and time consuming.

The efficiency of this process can be improved by conducting a sensitivity analysis in the early phase of data collection, on a model initially filled with rough estimates. The aim of this approach is to tend towards a cost efficient information enhancement - from a value of information optimization perspective. Indeed, SA conducted on a model incorporating rough estimate allow to roughly identify whether more accuracy is needed, i.e. if the alternatives have close expected values. When data is lacking and no elicitation is immediately possible, therefore in case of relative ignorance, the decision analyst can expand the variation range of the inputs under consideration and examine whether and how the inputs actually impact the decision. When conducting the sensitivity analysis with 
approximates (or rough estimates), the decision analysis process does not change in principle, it is only the data gathering effort that is more efficiently allocated.

While studying the implementation of IRDS in the company, the SA on approximates leverage effect has been useful when working on historical cases.

\section{Conclusion and future work}

This paper addressed the question of the difficulties associated with the uncertainty encountered when implementing IRDS. Based on an empirical study extended for the research presented in this paper, we observed that $5 \%$ to $20 \%$ of IRDS inputs are already available in the current decision dossiers. The qualitative data also provided can support the belief modeling for probabilities of success of the technical solutions proposed.

We proposed to elicit the data providers' beliefs by the free elicitation rule, considering the tradeoff between accuracy and time and effort that people are willing to commit. Some data are difficult to elicit, mostly the ones related to the scenario of failure and the time-related functions. Estimating these inputs requires to examine assumptions with the data providers and to solicit their experience, which may be uneven among individuals. However, this exercise encourages critical thinking and prompts participants to step back from their usual frame of reference.

In order to better allocate effort when collecting data for using IRDS, we proposed to perform sensitivity analyses on rough estimates. In this way, the decision analyst can identify the inputs that are decision sensitive and increase the accuracy sought by elicitation or further analyses immediately available. Further research need to be performed to assess under which conditions and how frequently the deterministic approach can be used.

As we conducted our research we tackled the questions of incorporating various experts' beliefs that are sometimes contradicting each other. IRDS prompts the data providers to communicate their beliefs explicitly, and this enables the others to discuss them and contribute their knowledge to update prior beliefs.

This research could be extended by exploring ways to facilitate the data collection process and communication between the three roles involved in the IRDS. Working on ongoing vehicle projects could benefit from the availability of people, as they would work in coherence with the activities for which they have been mandated. Considering an information system and practices supported by the management of the company would facilitate a more efficient use of IRDS.

\section{References}

Aladwani, A.M. (2001), “Change management strategies for successful ERP implementation”, Business Process Management Journal, Vol. 7 No. 3, pp. 266-275.

Blessing, L.T.M. and Chakrabarti, A. (2009), DRM, a Design Research Methodology, Springer London, London, available at: https://doi.org/10.1007/978-1-84882-587-1

Chen, W., Hoyle, C. and Wassenaar, H.J. (2013), Decision-Based Design, Springer London, London, available at: https://doi.org/10.1007/978-1-4471-4036-8

Hollard, G., Massoni, S. and Subjective, J.V. (2010), "Subjective beliefs formation and elicitation rules: experimental evidence HAL Id : halshs-00543828 Centre d'Economie de la Sorbonne Documents de Travail du Subjective beliefs formation and elicitation rules:"

Howard, R. and Abbas, A. (2015), Foundations of Decision Analysis, Prentice Hall, New-York.

Iooss, B. and Lemaître, P. (2015), "A Review on Global Sensitivity Analysis Methods", In: G., D. and C., M. (Eds.), Uncertainty Management in Simulation-Optimization of Complex Systems, Springer Boston, Boston, MA, pp. 101-122.

Jones, M.C., Cline, M. and Ryan, S. (2006), "Exploring knowledge sharing in ERP implementation: an organizational culture framework", Decision Support Systems, Vol. 41 No. 2, pp. 411-434.

Laskey, K.B. (1995), "Sensitivity analysis for probability assessments in Bayesian networks", IEEE Transactions on Systems, Man, and Cybernetics, Vol. 25 No. 6, pp. 901-909.

Leurent, B. et al. (2018), "Sensitivity Analysis for Not-at-Random Missing Data in Trial-Based CostEffectiveness Analysis: A Tutorial”, PharmacoEconomics, Vol. 36 No. 8, pp. 889-901.

Matt, C., Hess, T. and Benlian, A. (2015), "Digital Transformation Strategies", Business \& Information Systems Engineering, Vol. 57 No. 5, pp. 339-343. 
Rocquigny, E. de, Devictor, N. and Tarantola, S. (2008), Uncertainty in Industrial Practice: A Guide to Quantitative Uncertainty Management, edited by Rocquigny, E. de, Devictor, N. and Tarantola, S., Wiley \& Sons, Ltd.

Sissoko, T.M. (2019), Supporting Decision-Making for Solving Design Issues in the Development Phase of Automotive Vehicles, Université Paris-Saclay, CentraleSupélec.

Sissoko, T.M. et al. (2018), "An Empirical Study of a Decision-making Process Supported by Simulation in the Automotive Industry", Proceedings of the ASME 2018 International Design Engineering Technical Conferences \& Computers and Information in Engineering Conference.

Sissoko, T.M. et al. (2019), "A Proposal for a Decision Support Framework to Solve Design Problems in the Automotive Industry", Proceedings of the ASME 2019 International Design Engineering Technical Conferences and Computers and Information in Engineering Conference IDETC/CIE2019, August 18-21, 2019, Anaheim, CA, USA, pp. 1-10.

Thompson, S.C. and Paredis, C.J.J. (2010), “An Investigation Into the Decision Analysis of Design Process Decisions", Journal of Mechanical Design, Vol. 132 No. 12, p. 121009. 